\title{
Topology, the Wilson flow and the HMC algorithm
}

\author{
M. Lüscher* \\ CERN, Physics Department, 1211 Geneva 23, Switzerland \\ E-mail: Iuscher@mail.cern.ch
}

An old and apparently persistent problem in numerical lattice QCD is that the simulations tend to get trapped in a sector of fixed topological charge when the lattice spacing is taken to zero. The effect sets in very rapidly and may invalidate the simulation results in certain cases. In this talk, the issue is discussed using the Wilson flow as a tool. The flow has a simple scaling behaviour and allows one to understand how exactly the topological sectors emerge in the continuum limit. Further studies however suggest that the observed slowdown of the simulations at small lattice spacings is only partly caused by the emergence of the sectors.

The XXVIII International Symposium on Lattice Field Theory, Lattice2010

June 14-19, 2010

Villasimius, Italy

* Speaker. 


\section{Introduction}

At present all widely used simulation algorithms for lattice QCD with two or more flavours of sea quarks are based on some version of the HMC algorithm [1] (see ref. [2] for a recent review). The computer time required for such simulations depends on the time needed for an update step, the integrated autocorrelation times of the observables of interest and the desired statistics. As a function of the lattice spacing $a$, and if the algorithm is implemented as usual, the first factor scales roughly like $a^{-5}$, but the autocorrelation times tend to be difficult to determine and are often not known reliably if they are not small.

Large autocorrelation times are typically observed in the case of quantities related to the topological charge of the gauge field. While the definition of the charge on the lattice is ambiguous to some extent, experience suggests that the autocorrelation times are largely insensitive to the exact choices one makes. For illustration a time series of charge measurements is plotted in fig. 1. The presence of strong autocorrelations is evident in this example, particularly so in the shaded region, where the charge never changes sign and instead oscillates around a value of -10 . Note that most QCD simulations published to date are much shorter than the run shown in the figure.

The integrated autocorrelation times of physical quantities tend to grow when the continuum limit is approached. Depending on the theory, the algorithm and the observable, the asymptotic scaling behaviour can be very different, but is normally power-like in the inverse lattice spacing with an exponent $z \leq 2$. Del Debbio, Panagopoulos and Vicari [3] however found some time ago that the topological charge in the $\mathrm{SU}(3)$ gauge theory has a much more rapidly (perhaps exponentially) increasing autocorrelation time if the standard link-update algorithms are used. Further studies by Schaefer, Sommer and Virotta [ 4 ] later showed that the HMC algorithm is similarly inefficient and there is ample evidence that the problem persists when the sea quarks are added to the theory [ [ 6 -6].

In this talk, a few steps are taken towards a better understanding of the dynamics of the HMC algorithm and the mechanism that leads to the dramatic slowdown of the simulations at small lattice spacings. Issues to be addressed are how exactly the topological sectors emerge in the continuum limit, what the slow modes of the gauge field might be and whether perhaps there is a simple way out. The discussion is largely based on a new tool, the Wilson flow [7, 8], which is of some interest in its own right.

\section{Autocorrelations}

\subsection{Scaling behaviour}

Systematic scaling studies of autocorrelation times in lattice QCD have so far been limited to the case of the pure gauge theory [3, 可. In all these studies, the autocorrelation time of the topological charge turned out to increase at least like $a^{-5}$ at lattice spacings $a$ below $0.1 \mathrm{fm}$ or so. The algorithms considered (the HMC, the DD-HMC and the well-known link-update algorithms) appear to behave similarly in this respect. In particular, once the link-visiting frequency is divided out, the block size used in the DD-HMC algorithm does not have a significant influence on the autocorrelation times [4]. 


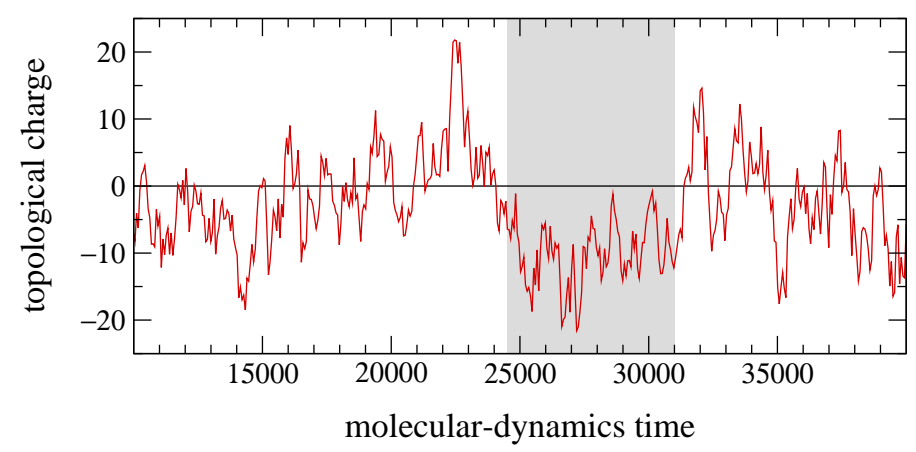

Figure 1: History of the topological charge in a simulation of the pure SU(3) gauge theory on a $64 \times 32^{3}$ lattice with spacing $a=0.07 \mathrm{fm}$. The plain HMC algorithm was used in this test run, with a trajectory length of 2 units of molecular-dynamics time and an acceptance rate of $83 \%$.

The onset of the rapid growth of the autocorrelation time of the topological charge, and thus its value at a given lattice spacing, however depends on the chosen lattice action and the simulation algorithm. Increasing the length of the molecular-dynamics trajectories in the HMC algorithm can be beneficial [П], for example, while the addition of six-link terms to the Wilson plaquette action may have an adverse effect [甘, 印.

When the sea quarks are included in the simulations, the situation becomes considerably more complicated, because the autocorrelations may now also depend on the number of quark flavours, the quark masses and chosen the fermion action [ [ 6 - These dependencies and the one on the lattice spacing remain to be studied in detail, but the experience made so far (which is sometimes only based on a visual inspection of measurement histories) shows that the autocorrelation time of the topological charge is again very rapidly growing when the lattice spacing is reduced from 0.1 $\mathrm{fm}$ to $0.05 \mathrm{fm}$ or even smaller values.

If the leading exponential autocorrelation time is assumed to grow proportionally to $a^{-5}$, the total computational effort required for HMC simulations of QCD is expected to scale like $a^{-10}$ at fixed physics, i.e. the cost of the simulations increases by about three orders of magnitude when the lattice spacing is divided by 2 . This estimate may be a bit pessimistic, but it is quite clear that the simulations required for safe extrapolations to the continuum limit are extremely challenging. Algorithmic improvements or viable ways of bypassing the slowing down of the simulations are certainly highly desirable at this point.

\subsection{Autocorrelation effects in short runs}

A question often asked in this context is whether the simulations really need to be very much longer than the leading exponential autocorrelation time. In particular, if the quantities of interest are only weakly coupled to the slow modes of the algorithm, the results obtained in shorter runs may conceivably be correct within statistical errors.

In general, the expectation values calculated in such short runs must be expected to be biased to some extent. Considering again the measurement history plotted in fig. 1. for example, it is clear that the topological charge is incorrectly sampled in this case if runs not very much longer than 5000 molecular-dynamics time units are performed. The expectation values of most observables are then affected by terms inversely proportional to the space-time volume [9, 10]. Physical quantities like 


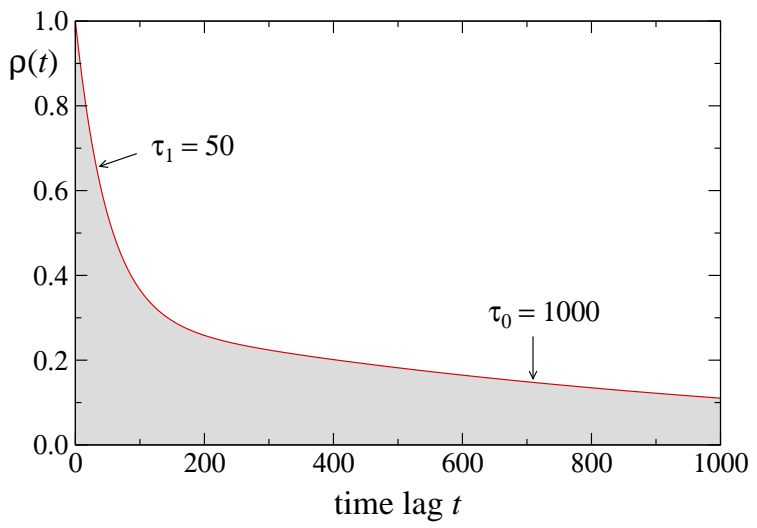

Figure 2: Example of a normalized autocorrelation function $\rho(t)$ that is dominated by two modes with exponential autocorrelation times $\tau_{0} \gg \tau_{1}$.

the masses of the $\eta$ and $\eta^{\prime}$ mesons, but also hadronic matrix elements of pseudo-scalar densities are likely to be strongly sensitive to these effects.

When arguing for short runs, one would need to provide a practical procedure that allows the effects of the slow modes on the calculated expectation values to be estimated. Whether they are in fact negligible in the cases of interest is otherwise difficult to tell and one is left with results that may or may not be correct.

\subsection{Statistical error estimation in presence of slow modes}

Large exponential autocorrelation times can also lead to an underestimation of integrated autocorrelation times and thus of the associated statistical errors. To illustrate this point, consider a normalized autocorrelation function

$$
\rho(t)=\left|c_{0}\right|^{2} \mathrm{e}^{-t / \tau_{0}}+\left|c_{1}\right|^{2} \mathrm{e}^{-t / \tau_{1}}+\ldots
$$

which is dominated by two eigenmodes of the simulation transition probability, a slow mode with exponential autocorrelation time $\tau_{0}$ and a fast mode with autocorrelation time $\tau_{1} \ll \tau_{0}$ (see fig. 2). The coefficients $\left|c_{0}\right|^{2}$ and $\left|c_{1}\right|^{2}$ in this formula measure how strongly these modes couple to the observable considered. Note that the integrated autocorrelation time

$$
\tau_{\text {int }} \simeq \tau_{0}\left|c_{0}\right|^{2}+\tau_{1}\left|c_{1}\right|^{2}+\ldots
$$

is equal to the area under the curve shown in the figure. Whether $\tau_{\text {int }}$ can be easily estimated now depends on whether the area under the tail of the curve dominates or not. If it does not, i.e. if $\tau_{0}\left|c_{0}\right|^{2} \ll \tau_{1}\left|c_{1}\right|^{2}$, the relevant exponential autocorrelation time is $\tau_{1}$ and the statistical error is correctly obtained from simulations a few hundred times longer than $\tau_{1}$. Runs very much longer than $\tau_{0}$ are however required in the other case to be able to control the situation.

Note that the contribution of the slow mode to the statistical error is not guaranteed to be negligible even if the coupling $\left|c_{0}\right|^{2}$ is very small. These cases are actually particularly difficult to treat correctly, because the autocorrelation function in the tail is very small, while the area under the tail may not be so. Since the autocorrelation function itself can only be calculated up to some statistical uncertainty, it may then be quite impossible to exclude this case, except when $\tau_{0}$ is known or can at least be bounded from above [ [ 


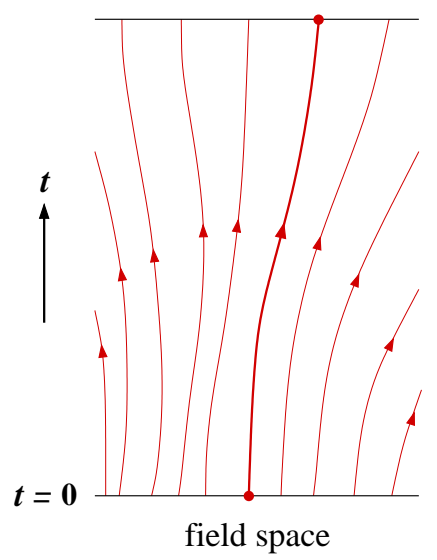

Figure 3: Equation (3.1) generates a flow in the space of lattice gauge fields. On a finite lattice, the existence, smoothness and uniqueness of the flow is rigorously guaranteed at all flow times [7]. Moreover, at any given time $t$, the mapping $U \rightarrow V_{t}$ is an invertible transformation of field space, because the flow can be integrated both forward and backward in time.

\section{Wilson flow}

The high-frequency components of the gauge field are usually weakly coupled to the slow modes of the HMC algorithm and tend to be efficiently updated. What exactly one means by the smooth components of the field is not entirely clear, however, and even less so which of their properties (apart from the topological charge) are slowly sampled by the algorithm. As explained in the following, the Wilson flow [7, 8] is a renormalizable smoothing operation that allows these questions to be addressed on theoretically solid ground.

\subsection{Flow equation}

For any given lattice gauge field $U(x, \mu)$, the first-order differential equation

$$
\dot{V}_{t}(x, \mu)=-g_{0}^{2}\left\{\partial_{x, \mu} S_{\mathrm{w}}\left(V_{t}\right)\right\} V_{t}(x, \mu),\left.\quad V_{t}(x, \mu)\right|_{t=0}=U(x, \mu),
$$

defines a trajectory $V_{t}(x, \mu)$ of fields parameterized by the "flow time" $t$ (see fig. 3; differentiation with respect to $t$ is abbreviated by a dot). In this equation, $S_{\mathrm{w}}\left(V_{t}\right)$ denotes the Wilson plaquette action [11] of the field $V_{t}$ at gauge coupling $g_{0}$ and $\partial_{x, \mu} S_{\mathrm{w}}\left(V_{t}\right)$ its (Lie algebra valued) variation with respect to the link variable $V_{t}(x, \mu)$. Note that the coupling cancels in the flow equation.

Along the Wilson flow, the plaquette action decreases monotonically, $\dot{S}_{\mathrm{w}} \leq 0$, and the gauge field tends to become smoother. The flow is in fact generated by infinitesimal "stout" link-smearing steps [12] and thus shares some properties with this popular smoothing procedure. Eventually the flow drives the field towards the stationary points of the action, but contrary to what may be assumed, the large-time regime is a highly non-perturbative one in QCD. In particular, it may not be meaningful to study the large-time behaviour of the flow separately from the continuum limit of the theory. 


\subsection{QED — a soluble case}

In the continuum limit, the flow equation (3.1) assumes the form

$$
\dot{B}_{\mu}=D_{\nu} G_{v \mu},\left.\quad B_{\mu}\right|_{t=0}=A_{\mu},
$$

where $A_{\mu}(x)$ is the fundamental gauge field, $B_{\mu}(t, x)$ the time-dependent gauge field and $G_{\mu \nu}(t, x)$ the associated field tensor. In QED the equation is a linear diffusion equation, whose solution is given by

$$
B_{\mu}(t, x)=\int \mathrm{d}^{4} y K_{t}(x-y) A_{\mu}(y)+\text { gauge terms, } \quad K_{t}(z)=\frac{\mathrm{e}^{-\frac{z^{2}}{4 t}}}{(4 \pi t)^{2}} .
$$

The flow thus averages the gauge field over a spherical range with mean-square radius equal to $\sqrt{8 t}$. Note that, as is already clear from eq. (3.2), the flow time $t$ has engineering dimension [length $]^{2}$.

Since the field generated by the flow is linearly related to the fundamental gauge field, its correlation functions

$$
\begin{aligned}
\left\langle B_{\mu_{1}}\left(t, x_{1}\right) \ldots B_{\mu_{n}}\left(t, x_{n}\right)\right\rangle=e_{0}^{n} \int & \mathrm{d}^{4} y_{1} \ldots \mathrm{d}^{4} y_{n} K_{t}\left(x_{1}-y_{1}\right) \ldots K_{t}\left(x_{n}-y_{n}\right) \\
& \times G_{0}\left(y_{1}, \ldots, y_{n}\right)_{\mu_{1} \ldots \mu_{n}}+\text { gauge terms }
\end{aligned}
$$

are proportional to the (full, bare) photon $n$-point functions $G_{0}\left(y_{1}, \ldots, y_{n}\right)_{\mu_{1} \ldots \mu_{n}}$. A power of the bare electron charge $e_{0}$ appears in this equation, because the canonically normalized photon field is $e_{0} A_{\mu}$ rather than $A_{\mu}$ (which is normalized so that the covariant derivatives do not involve the charge). Note also that the heat kernels $K_{t}\left(x_{k}-y_{k}\right)$ play the rôle of smooth test functions in this formula. In particular, the renormalization of the correlation function is achieved simply by renormalizing the bare charge and the photon $n$-point function according to

$$
e_{0}=Z_{3}^{-1 / 2} e_{\mathrm{R}}, \quad G_{0}=Z_{3}^{n / 2} G_{\mathrm{R}} .
$$

The fact that both $e_{0}$ and $G_{0}$ renormalize with the same renormalization constant $Z_{3}$ is a consequence of the gauge Ward identity in this theory (a gauge-invariant regularization is assumed here). Since $e_{0}^{n} G_{0}=e_{\mathrm{R}}^{n} G_{\mathrm{R}}$, this shows that the field $B_{\mu}(t, x)$ is, at all positive flow times $t$ and up to its gauge degrees of freedom, a renormalized smooth gauge field.

Provided the bare charge is expressed through the renormalized one, the correlation functions of the field tensor $G_{\mu \nu}$ thus do not require any renormalization and converge to well-defined smooth functions of the space-time coordinates when the regularization of the theory is removed. Moreover, since QED is asymptotically free at low energies, the behaviour of the correlation functions at large flow times is described by leading-order perturbation theory. A short calculation then leads to the formula

$$
\lim _{t \rightarrow \infty}\left\{t^{2}\left\langle G_{\mu v} G_{\mu v}\right\rangle\right\}=\frac{3 e_{\mathrm{R}}^{2}}{32 \pi^{2}},
$$

which shows that the field obtained by the Wilson flow contains some interesting physical information. 


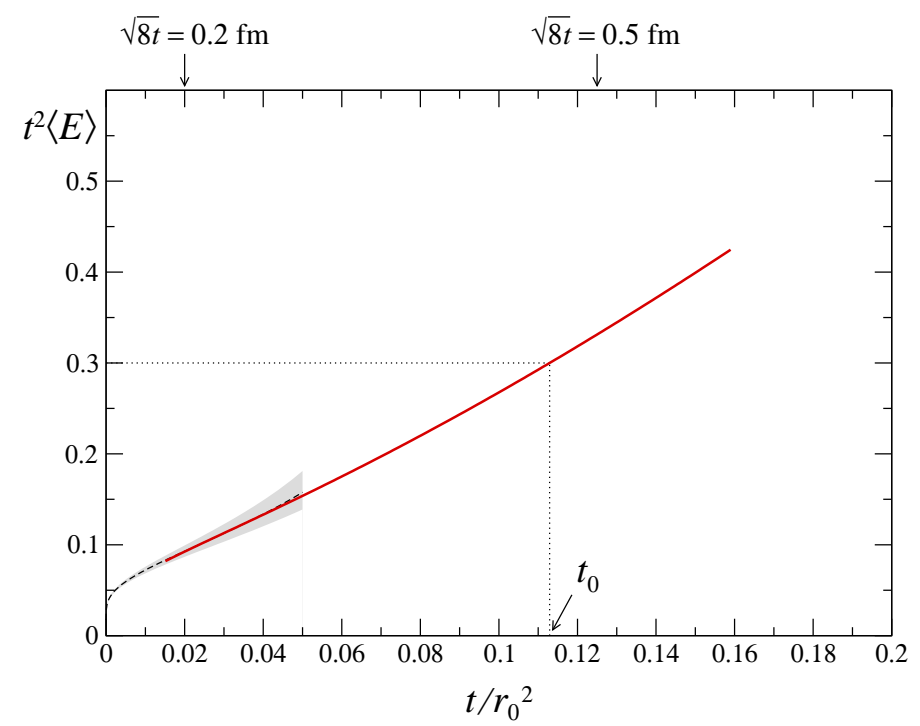

Figure 4: Plot of $t^{2}\langle E\rangle$ in the pure SU(3) gauge theory as a function of the flow time $t$ given in units of the Sommer scale $r_{0}[13,14]$. The thick solid line was obtained through numerical simulation of a $96 \times 48^{3}$ lattice with spacing $a=0.05 \mathrm{fm}$. One-loop perturbation theory [eq. (3.8)] and the known value of the $\Lambda$ parameter [15] yield the dashed curve with an error margin (grey area) deriving from the error on $r_{0} \Lambda$.

\subsection{Properties of the Wilson flow in QCD}

In QCD the flow equation is non-linear and the renormalization of the theory is much more complicated than in QED. Whether the Wilson flow generates a renormalized gauge field is however a question that can be studied in perturbation theory. In particular, the expectation value of the gauge-invariant density

$$
E=-\frac{1}{2} \operatorname{tr}\left\{G_{\mu \nu} G_{\mu \nu}\right\}
$$

can be easily worked out to next-to-leading order in the gauge coupling. Dimensional regularization may be used in this calculation and it then turns out that $\langle E\rangle$ does not require renormalization to this order, i.e. the divergent terms are all canceled by the renormalization of the coupling. In the $\overline{\mathrm{MS}}$ scheme, the one-loop formula obtained in this way is [8]

$$
\begin{aligned}
\langle E\rangle & =\frac{3}{4 \pi t^{2}} \alpha(q)\left\{1+k_{1} \alpha(q)+\ldots\right\}, \quad q=(8 t)^{-1 / 2}, \\
k_{1} & =1.0978+0.0075 \times N_{\mathrm{f}},
\end{aligned}
$$

where $N_{\mathrm{f}}$ denotes the number of massless sea quarks and the running coupling $\alpha(q)$ is evaluated at a momentum scale $q$ equal to the inverse of the leading-order smoothing range of the flow.

Beyond perturbation theory, the expectation value of $E$ can be computed straightforwardly using the lattice formulation of the theory and numerical simulations. An accurate numerical integration of the flow equation (3.1) is required in these calculations, but the computer time needed for the integration is negligible in practice if a suitable higher-order integrator is used [8]. The result of a computation along these lines is plotted in fig. $\forall$ in a range of the flow time corresponding to smoothing ranges from about 0.2 to $0.5 \mathrm{fm}$ (the statistical errors are not visible on the scale of the 


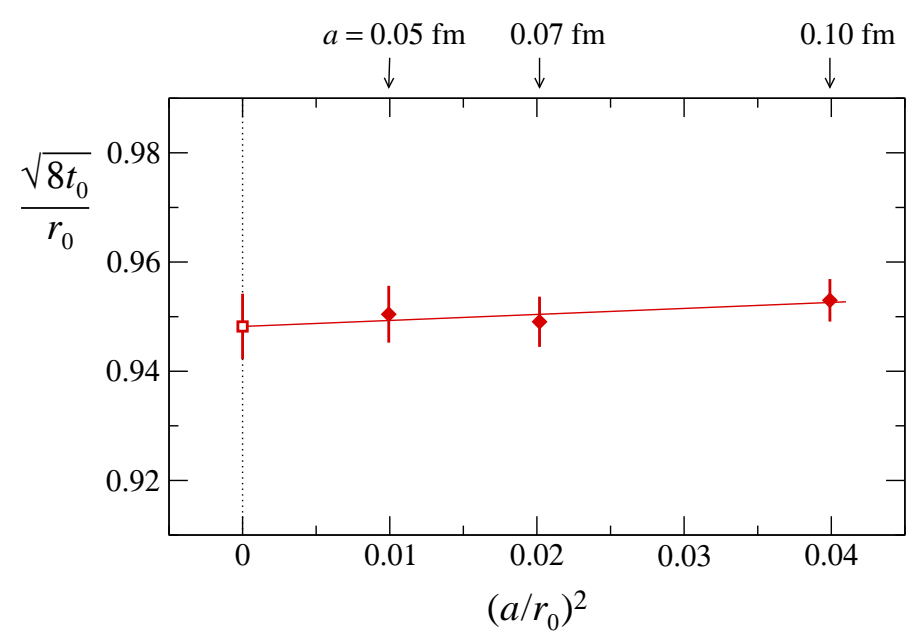

Figure 5: Scaling behaviour of the reference time $t_{0}$ in the pure SU(3) gauge theory. The data (full points) were obtained on lattices of size $48 \times 24^{3}, 64 \times 32^{3}$ and $96 \times 48^{3}$ with spacings approximately equal to 0.1 , 0.07 and $0.05 \mathrm{fm}$, respectively. The continuum limit (open point) is reached through a linear fit in $a^{2}$ of the three data points (solid line).

plot). Since QCD is asymptotically free, the perturbation expansion (3.8) only applies at small flow times, but as can be seen from the curves shown in fig. $\sharp$, the transition from the small-time to the non-perturbative regime is very smooth. Note that $t^{2}\langle E\rangle$ increases roughly linearly with $t$ in the non-perturbative regime, at least so in the range shown in the plot, a behaviour which is completely different from the one in QED.

If $\langle E\rangle$ does not require renormalization, one expects $t^{2}\langle E\rangle$ to be a universal function of $t / r_{0}^{2}$ up to lattice effects that vanish proportionally to a positive power of the lattice spacing. The scaling can be checked by introducing a reference time $t_{0}$ through the implicit equation

$$
\left.t^{2}\langle E\rangle\right|_{t=t_{0}}=0.3
$$

(see fig. (4). Simulations at three values of the lattice spacings then show that the dimensionless ratio $\sqrt{8 t_{0}} / r_{0}$ does in fact smoothly converge to the continuum limit, the lattice-spacing effects being less than a percent in the range covered by the simulations (fig. 5). Little doubt thus remains that the Wilson flow maps the gauge field to a renormalized smooth field as in QED.

\section{Topological sectors}

The question of how exactly the topological sectors emerge in lattice QCD can now be answered by performing the field transformation $U \rightarrow V=V_{t_{0}}$ in the functional integral, $t_{0}$ being the reference flow time introduced in the previous section. The smoothing range is about $0.5 \mathrm{fm}$ in this case, i.e. on average the fluctuations of the gauge field with wavelengths up to the confinement radius are smoothed out. Somewhat surprisingly, the Jacobian of the transformation can be analytically expressed through the Wilson action [7]. The expectation value of any observable $\mathscr{O}(U)$ then assumes the form

$$
\langle\mathscr{O}\rangle=\frac{1}{\mathscr{Z}} \int \mathrm{D}[V] \mathscr{O}(U) \mathrm{e}^{-\tilde{S}(V)}
$$




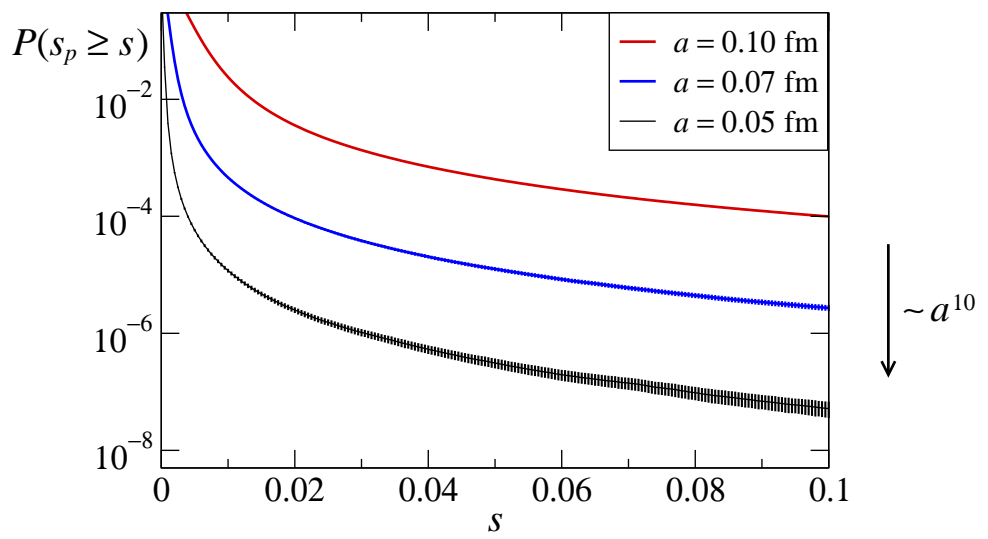

Figure 6: Probability for the value of $s_{p}$ [eq. (4.3)] on a given plaquette $p$ to be above a specified threshold $s$. The three curves were obtained in the pure SU(3) gauge theory with Wilson plaquette action, using the same representative ensembles of gauge-field configurations as in the scaling test reported in fig. 5.

where the action is given by

$$
\tilde{S}(V)=S(U)+\frac{16 g_{0}^{2}}{3 a^{2}} \int_{0}^{t_{0}} \mathrm{~d} t S_{\mathrm{w}}\left(V_{t}\right) .
$$

In this formula, the fields $U$ and $V_{t}$ are considered to be functions of $V$ and $S(U)$ denotes the action of the theory (including the quark determinants if any) before the transformation.

Both terms in the action (4.2) tend to suppress large values of the plaquette observable

$$
s_{p}=\operatorname{Re} \operatorname{tr}\{1-V(p)\}
$$

(where $V(p)$ is the product of the link variables $V(x, \mu)$ around the plaquette $p$ ) and thus force the plaquette loops to be close to unity. Since $s_{p}$ is a field of dimension 4 , representing the square of the gauge-field tensor in the plaquette plane, its expectation value is in fact expected to scale like $a^{4}$ in the continuum limit. Numerical studies confirm this behaviour and show that large values of $s_{p}$ are indeed very strongly suppressed (see fig. 6).

Many years ago, a theorem was established [16, 17] which states that the space of all lattice gauge fields satisfying a certain smoothness condition decomposes into topological charge sectors very much like the space of continuous fields in the classical continuum theory. In particular, the theorem applies to the subspace of $\mathrm{SU}(3)$ gauge fields $V$ which satisfy

$$
s_{p}<0.067 \text { for all plaquettes } p \text {. }
$$

According to the empirical results reported in fig. 6, and since the number of plaquettes in a fixed physical volume grows proportionally to $a^{-4}$, the fields that do not fulfill this condition occur with a probability of order $a^{6}$. In the functional integral (4.1), the weight of these configurations (and thus of the region of field space, where the assignment of the topological charge is ambiguous) is therefore rapidly decreasing when the lattice spacing is taken to zero. In lattice gauge theory, the emergence of the topological sectors is thus seen to be a dynamical phenomenon, which sets in close to the continuum limit. 


\begin{tabular}{cccc}
$t / t_{0}$ & $\tau_{\text {int }}[Q]$ & $\tau_{\text {int }}\left[Q^{2}\right]$ & $\tau_{\text {int }}[E]$ \\
\hline \hline 0.18 & $65(5)$ & $30(2)$ & $25(2)$ \\
0.35 & $67(5)$ & $32(2)$ & $35(2)$ \\
0.70 & $68(6)$ & $33(2)$ & $44(3)$ \\
\hline \hline
\end{tabular}

\begin{tabular}{cccc}
$t / t_{0}$ & $\tau_{\text {int }}[Q]$ & $\tau_{\text {int }}\left[Q^{2}\right]$ & $\tau_{\text {int }}[E]$ \\
\hline \hline 0.18 & $614(90)$ & $284(34)$ & $55(4)$ \\
0.36 & $615(90)$ & $286(34)$ & $69(5)$ \\
0.72 & $615(90)$ & $286(34)$ & $86(6)$ \\
\hline \hline
\end{tabular}

Table 1: Integrated autocorrelation times in the pure SU(3) gauge theory with Wilson action, measured on a $48 \times 24^{3}$ lattice with spacing $a=0.1 \mathrm{fm}$ (left table) and on a $64 \times 32^{3}$ lattice with spacing $a=0.07 \mathrm{fm}$ (right table). In both cases, the HMC algorithm with a trajectory length of 2 and an acceptance rate of $83 \%$ was used. All autocorrelation times and the trajectory length are given in units of molecular-dynamics time.

\section{Slow modes of the HMC algorithm}

The field transformation considered in the previous section maps the molecular-dynamics trajectories generated by the HMC algorithm to trajectories of the transformed field $V=V_{t_{0}}$. At lattice spacings where the regions of field space "between the topological sectors" are strongly suppressed, it is unlikely that such a trajectory leads from one sector to another, because the fields along the trajectories are, to a good approximation, distributed according to their weight in the functional integral. The emergence of the sectors and the freezing of the topological charge in HMC simulations are thus directly related to each other.

\subsection{Do the smoothed fields move slowly?}

The algorithm may however slow down for other reasons as well and there is no guarantee that the gauge field is efficiently updated in a fixed sector. In particular, the smooth fields obtained by the Wilson flow at flow time $t>0$ may conceivably be slowly moved through configuration space, in which case the autocorrelation times of local observables constructed from these fields (such as $E)$ are expected to be large.

The measured autocorrelation times of $E$ listed in table 1 are actually monotonically increasing with the flow time $t$ and reach values up to 10 times the autocorrelation time of the usual plaquette observable at $t=0$. On the other hand, when the lattice spacing is reduced, they do not appear to grow as rapidly as the autocorrelation times of the topological charge $Q$ (in these studies, $E$ and the charge density were both defined using the same symmetric plaquette-loop expression for the gauge-field tensor at the specified flow time). The relatively slow updating of the smoothed field and the charge freezing are thus two different effects, where one is not obviously driving the other.

\subsection{Open boundary conditions}

One may be inclined to conclude at this point that the emergence of the topological sectors at small lattice spacings is the principal and perhaps only cause for the presence of very rapidly growing autocorrelation times in HMC simulations. However, when choosing open instead of periodic boundary conditions in the physical time direction, the topological sectors disappear and the space of smooth fields becomes connected. One thus expects to observe only moderately increasing autocorrelation times in this case if the slowdown of the algorithm is indeed mainly caused by the separation of the sectors. 


\begin{tabular}{lccc} 
& $\tau_{\text {int }}[Q]$ & $\tau_{\text {int }}\left[Q^{2}\right]$ & $\tau_{\text {int }}[E]$ \\
\hline \hline periodic & $68(6)$ & $33(2)$ & $44(3)$ \\
open & $61(6)$ & $27(2)$ & $38(3)$ \\
\hline \hline
\end{tabular}

\begin{tabular}{lccc} 
& $\tau_{\text {int }}[Q]$ & $\tau_{\text {int }}\left[Q^{2}\right]$ & $\tau_{\text {int }}[E]$ \\
\hline \hline periodic & $615(90)$ & $286(34)$ & $86(6)$ \\
open & $384(56)$ & $155(20)$ & $77(6)$ \\
\hline \hline
\end{tabular}

Table 2: Comparison of autocorrelation times measured on lattices with periodic and open boundary conditions in the physical time direction. The lattices are the same as in table 1 (left: $a=0.1 \mathrm{fm}$, right: $a=0.07$ $\mathrm{fm})$, the HMC parameters are also the same and the flow time $t$ is equal to $0.7 \times t_{0}$ in all cases.

A lattice with time extent $T$ and open boundary conditions in time does not wrap around in this direction, i.e. there are no terms in the action which couple the field variables at time $x_{0}=0$ to those at the largest time $x_{0}=T-a$. In a simulation program for periodic lattices, these boundary conditions can often be implemented simply by setting the time-like link variables at the largest time to zero. Note that open boundary conditions preserve the gauge symmetry. In the continuum theory, they amount to imposing Neuman boundary conditions,

$$
\left.F_{0 k}(x)\right|_{x_{0}=0}=\left.F_{0 k}(x)\right|_{x_{0}=T}=0, \quad k=1,2,3,
$$

on the gauge field and homogeneous Dirichlet (Schrödinger functional) boundary conditions on the quark fields, $F_{\mu \nu}$ being the field tensor of the fundamental gauge field. The field space becomes topologically trivial when these boundary conditions are chosen. In particular, instantons can be smoothly moved in and out of the volume through the boundaries at $x_{0}=0, T$.

The figures listed in table 2 show that the situation is in fact somewhat improved when passing from periodic to open boundary conditions (in both cases, $Q$ denotes the sum of the topological charge density over all lattice points). As a function of the lattice spacing, the autocorrelation time of $Q$ is however rapidly growing independently of the chosen boundary condition. On the periodic lattices, the quantization of the topological charge thus appears to be only one of several causes of the slowdown of the HMC algorithm.

\subsection{Instantons and the chiral limit}

One of the distinguishing features of non-abelian gauge theories is the existence of the instanton solutions of the classical field equations. The fact that the action has a large manifold of nearly stationary points (the configurations built from many distant instantons and anti-instantons) may conceivably play an important dynamical rôle in these theories, but so far it proved to be difficult to actually show this. In the present context, the observation is perhaps of some relevance, because the fields generated by the Wilson flow at large flow times tend to be close to the stationary points of the action.

In QCD with light sea quarks, the fluctuations of the topological charge are suppressed and the smooth fields obtained by the Wilson flow may therefore be characteristically different from the ones in the pure gauge theory. The slow modes of the HMC algorithm consequently need not be the same and the autocorrelation times of the usual quantities may turn out to have a significant dependence on the quark masses. However, as already mentioned, systematic studies of autocorrelation times await to be performed in QCD with dynamical quarks. 


\section{Conclusions}

Numerical lattice QCD rests on the assumption that simulations at sufficiently small lattice spacings can eventually be performed to be able to control the discretization errors. It is therefore of central importance to overcome the poor scaling behaviour of the currently available simulation algorithms. Finding better algorithms is difficult, however, and probably requires a more detailed understanding of why the HMC algorithm slows down when the lattice spacing is taken to zero.

The Wilson flow is a useful tool in this context, because it allows the properties of the gauge field at different length scales to be studied consistently with the renormalization of the theory. In particular, the way in which the topological sectors emerge close to the continuum limit is made transparent through the flow. The emergence of these sectors is certainly one of the principal causes of the slowdown of the HMC algorithm, but the fact that the situation improves only slightly on lattices with open boundary conditions is a bit surprising and remains unexplained. It seems safe to conclude, however, that there are important further sources of inefficiency and that the problem is likely to persist at fixed topological charge (the rapid slowdown of the algorithm is in this case expected to be revealed when considering the sum of the charge density over half the lattice).

Choosing open boundary conditions in the physical time direction is nevertheless an interesting option, because the barriers between the sectors disappear in this case, while the transfer matrix (and thus much of the physics) is the same as with periodic boundary conditions. From the point of view of the simulation algorithms, the absence of the barriers represents an important simplification. Tunneling transitions are then not required anymore and one is left with the task of finding an algorithm that moves the field $V_{t}$ (at, say, $t=t_{0}$ ) efficiently through the space of smooth configurations.

\section{Acknowledgments}

I am indebted to Filippo Palombi, Stefan Schaefer and Rainer Sommer for useful discussions on various issues related to this talk. All numerical simulations reported here were performed on a dedicated PC cluster at CERN. I am grateful to the CERN management for providing the required funds and to the CERN IT Department for technical support.

\section{References}

[1] S. Duane, A. D. Kennedy, B. J. Pendleton, D. Roweth, Hybrid Monte Carlo, Phys. Lett. B195 (1987) 216.

[2] M. Lüscher, Computational strategies in lattice QCD, Lectures given at the Summer School on “Modern perspectives in lattice QCD”, Les Houches, August 3-28, 2009, arXiv:1002.4232 [hep-lat].

[3] L. Del Debbio, H. Panagopoulos, E. Vicari, $\theta$-dependence of $S U(N)$ gauge theories, JHEP 08 (2002) 044.

[4] S. Schaefer, R. Sommer, F. Virotta, Investigating the critical slowing down of QCD simulations, PoS (LAT2009) 032; Critical slowing down and error analysis in lattice QCD simulations, arXiv:1009.5228 [hep-lat]. 
[5] D. J. Antonio et al. (RBC and UKQCD Collab.), Localization and chiral symmetry in 2+1 flavor domain wall QCD, Phys. Rev. D77 (2008) 014509.

[6] A. Bazavov et al. (MILC Collab.), Topological susceptibility with the asqtad action, Phys. Rev. D81 (2010) 114501.

[7] M. Lüscher, Trivializing maps, the Wilson flow and the HMC algorithm, Commun. Math. Phys. 293 (2010) 899 .

[8] M. Lüscher, Properties and uses of the Wilson flow in lattice QCD, JHEP 08 (2010) 071.

[9] R. Brower, S. Chandrasekharan, J. Negele, U.-J. Wiese, QCD at fixed topology, Phys. Lett. B560 (2003) 64.

[10] S. Aoki, H. Fukaya, S. Hashimoto, T. Onogi, Finite-volume QCD at fixed topological charge, Phys. Rev. D76 (2007) 054508.

[11] K. G. Wilson, Confinement of quarks, Phys. Rev. D10 (1974) 2445.

[12] C. Morningstar, M. Peardon, Analytic smearing of $S U(3)$ link variables in lattice QCD, Phys. Rev. D69 (2004) 054501.

[13] R. Sommer, A new way to set the energy scale in lattice gauge theories and its applications to the static force and $\alpha_{s}$ in SU(2) Yang-Mills theory, Nucl. Phys. B411 (1994) 839.

[14] M. Guagnelli, R. Sommer, H. Wittig (ALPHA collab.), Precision computation of a low-energy reference scale in quenched lattice QCD, Nucl. Phys. B535 (1998) 389.

[15] S. Capitani, M. Lüscher, R. Sommer, H. Wittig (ALPHA collab.), Non-perturbative quark mass renormalization in quenched lattice QCD, Nucl. Phys. B544 (1999) 669.

[16] M. Lüscher, Topology of lattice gauge fields, Commun. Math. Phys. 85 (1982) 39.

[17] A. Phillips, D. Stone, Lattice gauge fields, principal bundles and the calculation of the topological charge, Commun. Math. Phys. 103 (1986) 599. 